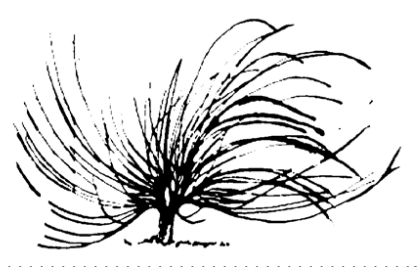

\title{
Cultura, emociones y aprendizaje significativo en Educación Física
}

\author{
Felipe Nicolás Mujica-Johnson ${ }^{1}$ \\ Facultad de Educación \\ Universidad Autónoma de Chile \\ Chile \\ felipe.mujica@uautonoma.cl
}

\begin{abstract}
José Ignacio Salgado-López ${ }^{2}$
Instituto de Educación Secundaria Leliadoura

Consellería de Educación, Universidade e Formación Profesional (Xunta de

Galicia)

España

inhaqui@edu.xunta.gal
\end{abstract}

\begin{abstract}
Resumen
En las últimas décadas se está desarrollando un giro afectivo en la teoría y práctica de los entornos pedagógicos, lo que ha generado que las emociones tengan mayor importancia en el aprendizaje escolar. En ese contexto educativo se ha elaborado este ensayo, que tiene por objetivo exponer la relación de los significados culturales con el aprendizaje significativo en la asignatura de Educación Física en función de la perspectiva emocional. Los análisis estuvieron centrados, principalmente, en los significados asociados al género, el nivel socioeconómico y el
\end{abstract}

Recibido: 11 de setiembre de 2020. Aprobado: 1 de junio de 2021

http://dx.doi.org/10.15359/rep.16-2.4

1 Magíster en Ciencias de la Actividad Física y del Deporte, Universidad de Playa Ancha de Ciencias de la Educación, Chile. Doctorando en Ciencias de la Actividad Física y del Deporte, Universidad Politécnica de Madrid, España. Profesor asociado del Centro de Investigación Escolar y Desarrollo, Universidad Católica de Temuco, Chile. ORCID: https://orcid. org/0000-0002-6956-2357

2 Doctor en Educación Física, Universidade da Corunha, Galiza - España. Funcionario de Carrera del Cuerpo de Profesores de Enseñanza Secundaria, especialidad de Educación Física. Profesor Colaborador LOU de la Universidad de Zaragoza en excedencia. ORCID: https:// orcid.org/0000-0002-6561-6450 
contacto como manifestaciones culturales. Se describen diferentes estudios que explican la influencia de dichos aspectos en las emociones de los estudiantes durante su aprendizaje motor. Estos significados pueden alterar la disposición emocional del alumnado ante el aprendizaje de nuevas conductas motrices, por lo que el aprendizaje motor significativo también se vería afectado. No obstante, según dicha teoría, la mala disposición emocional no impediría desarrollar nuevas conductas motrices. Pero, sería importante que el profesorado tenga una buena comprensión de las posibilidades motrices de cada estudiante para optimizar el proceso y no influir negativamente en su bienestar subjetivo. Se concluye, que ante los significados culturales que promuevan emociones displacenteras ante algún contenido didáctico, sería muy útil promover la adquisición de nuevos significados, que tengan una orientación contraria, para mejorar la disposición emocional de los estudiantes.

Palabras clave: Afectividad, aprendizaje motor, cultura, educación física, subjetividad

\begin{abstract}
In the last decades, an affective shift has been developing in the theory and practice of pedagogical environments, which has caused emotions to be of greater importance in school learning. In this educational context, this essay has been elaborated with the objective of describing the relationship of cultural meanings with meaningful learning in the Physical Education subject based on an emotional perspective. The analyzes were mainly focused on the meanings associated with gender, socioeconomic status, and contact as cultural manifestations. Different studies are described that explain the influence of these aspects on the emotions of students during their motor learning. These meanings can alter the emotional disposition of students when learning new motor behaviors, so significant motor learning would also be affected. However, according to this theory, poor emotional disposition would not prevent developing new motor behaviors. Still, it would be important for teachers to have a good understanding of the motor capabilities of each student to optimize the
\end{abstract}


process and not influence their subjective well-being negatively. It is concluded that in the face of cultural meanings that promote unpleasant emotions before some didactic content, it would be very useful to promote the acquisition of new meanings that have a contrary orientation to improve the emotional disposition of students.

Keywords: affectivity, culture, motor learning, physical education, subjectivity

\section{Introducción}

$\mathrm{E}$

$\mathrm{n}$ el marco del giro afectivo que se viene desarrollando en los últimos años en el ámbito educativo (Bozalek y Zembylas, 2016; Zembylas, 2019), la consideración de las emociones en el proceso de enseñanza-aprendizaje se hace cada vez más común en las discusiones pedagógicas (Mujica et al., 2019). Esta tendencia también se ha manifestado en el campo de Educación Física (EF), marcada por diferentes perspectivas, entre las que destacan, la educación emocional, la psicología positiva, la praxiología motriz, las teorías constructivistas y las teorías sociocríticas (Lagardera, 1999; Miralles, Filella y Lavega, 2017; Monforte y Pérez-Samaniego, 2017; Mujica-Johnson y Jiménez, 2020; Mujica y Orellana, 2019; Parlebas, 1970, 2017; Sáez de Ocáriz et al., 2014; Salgado-López, 2014, 2020; Timken et al., 2019). No obstante, en distintas revisiones bibliográficas sobre esta temática (Bermúdez y Sáenz-López, 2019; Espoz-Lazo et al., 2020; Mujica, 2019; Mujica y Orellana, 2019; Mujica et al., 2017), se puede apreciar desde nuestro punto de vista que, para ser un tema tan transversal e importante para la formación humana, tiene muchos aspectos que requieren ser más discutidos e investigados. Uno de ellos corresponde al factor cultural, reconocido por incidir en la subjetividad humana y, por ende, en su afectividad, desde el nacimiento (Lévi-Strauss, 2019), a través de las variadas estructuras sociales (Foucault, 2019).

En cuanto al término cultura, hemos de señalar que en las diferentes épocas históricas han existido múltiples formas de interpretarlo (Ron, 1977), de las cuales destacan en la época contemporánea los aportes de Lévi-Strauss $(1971,2019)$. En concreto, dicho autor señaló que cultura "... es el conjunto de las costumbres, creencias, institucionales tales como el arte, el derecho, la religión, técnicas de la vida material, en una 
palabra, todos los hábitos o aptitudes adquiridos por el hombre como miembro de una sociedad" (Lévi-Strauss, 1971, p. 131). En esta misma línea, Altieri (2001) ha planteado lo siguiente sobre el concepto cultura:

En él están comprendidos tanto el lenguaje, la industria, el arte, la ciencia, el derecho, el gobierno, la moral, la religión, como los instrumentos materiales o artefactos en los que se materializan las realizaciones culturales y mediante los cuales surten efecto práctico los aspectos intelectuales de la cultura (edificios, instrumentos, máquinas, objetos de arte, medios para la comunicación, etcétera). (p. 15)

Como se ha expresado anteriormente, son múltiples los temas que pueden ser relacionados con el ámbito cultural, lo cual ha dado vida a diferentes expresiones que intentan representar rasgos de ciertos grupos de la sociedad, tanto en el nivel micro y microsocial. En el nivel microsocial, podríamos hacer referencia a los aspectos culturales asociados a un grupo reducido de personas. En el macrosocial, podemos destacar modos de vida que representan a un amplio grupo de personas, por ejemplo, la cultura occidental, oriental, musulmana, cristiana, patriarcal, feminista, aristocrática, comunista, democrática, capitalista, entre otras (Altieri, 2001; Ron, 1977; Scheler, 2010; Lévi-Strauss, 2019). Cada una de dichas culturas presentaría su propia cosmovisión que, a su vez, disputaría en la sociedad la legitimidad de los valores que promueve. Además, las múltiples categorías culturales pueden ser enlazadas de diferente forma, lo cual también refleja la relativa complejidad en torno al estudio de los significados culturales.

Una conceptualización del término emoción que refleja, adecuadamente, el rol de la cultura es la de Puig (2012), quien señala que no se reduce a "una simple reacción fisiológica, sino que, a pesar de tener una dimensión de este tipo, también está relacionada con rasgos de personalidad (dimensión psicológica), subculturas específicas (modalidades deportivas -en nuestro caso-, género, edad...) y contextos socioculturales e históricos" (p. 106).

Dentro de las teorías del aprendizaje, cabe destacar las contribuciones de Piaget (1991), Vygotsky (1995), Ausubel (2002) y Bruner (1997), las cuales aportan visiones que reconocen la importancia de las experiencias socioculturales y su conexión con la afectividad. Para 
este ensayo, se colocará el foco en la teoría del aprendizaje significativo de Ausubel, la cual considera a las experiencias y los saberes previos de los sujetos como un aspecto fundamental para la adquisición de un nuevo aprendizaje. Por supuesto, ambos aspectos no son ajenos al contexto histórico-cultural. En este sentido, dicha teoría "enfatiza la necesidad de desarrollar una educación integral en el aula educativa, donde lo emocional y lo cognitivo sean igual de importantes" (Mujica y Orellana, 2019, pp. 304-305). Así, las experiencias previas positivas, junto a sus aprendizajes, se presentan como facilitadoras del aprendizaje y, al contrario, las experiencias negativas podrían obstaculizarlo (Cáceres y Munévar, 2016; Gea-García et al., 2016; Lavega et al., 2011; Parlebas, 2017; Salgado-López, 2020; Viera, 2003). Esta relación entre factores culturales, emociones y aprendizaje del alumnado es bastante más compleja de lo que puede parecer, ya que muchos de los significados implícitos en la configuración subjetiva-emocional de cada persona (González, 2009), pueden ser difíciles de ser reconocidos tanto por el profesorado como por el propio alumnado. Por otro lado, también podrían existir dificultades para interpretar dichos significados (Heinemann y Puig, 1997; Salgado-López, 2014). En este sentido, la comprensión de dicha correspondencia se puede hacer más difusa cuando comienza a interactuar con nuevos significados asociados a la disciplina pedagógica de EF como, por ejemplo, sus contenidos didácticos y equipamientos; las relaciones interpersonales con los pares y el profesorado, mediadas por estereotipos sociales; la infraestructura o espacio donde se desarrolla la sesión; y otros aspectos curriculares, como la metodología de enseñanza o las evaluaciones aplicadas.

Con base en lo expuesto con anterioridad, este ensayo tiene por objetivo, en primer lugar, presentar algunos factores culturales que pueden incidir en la vivencia emocional de estudiantes en el contexto de EF. Después, se analiza la relación de las emociones con la teoría del aprendizaje significativo (AS). Por último, se discute la relación de los significados culturales con el aprendizaje motor significativo (AMS) en EF desde la perspectiva emocional. Asimismo, se plantean algunas aplicaciones prácticas para que el profesorado pueda incluir las emociones en su propuesta didáctica considerando el factor cultural, con la finalidad de optimizar el aprendizaje del alumnado escolar. 


\section{Cultura y emociones en Educación Física}

El primer aspecto cultural que será abordado es el de la desigualdad de género en función de la histórica tradición machista y patriarcal, según la cual se estableció la dominación masculina en la sociedad (Bourdieu, 2000). La EF no ha sido aséptica a esta influencia, al ser uno de los espacios pedagógicos que ha visto encarnada dicha costumbre con múltiples manifestaciones. De ellas destacan los estereotipos de género que asocian a las chicas, entre otras características, con la estética y la debilidad, mientras a los chicos, con la inteligencia y la fortaleza (Alvariñas et al., 2009; Camacho-Miñano, 2013; Gaviria y Castejón, 2016). Esta mirada patriarcal de la masculinidad y la feminidad en EF, generaría un ambiente hostil para estudiantes que no se sientan identificados con ella, pudiendo, incluso, llegar a sentirse presionados por dichos estereotipos. Un ejemplo de lo anterior se ve reflejado en el estudio en educación secundaria realizado en Nueva Zelanda (Gerdin y Larsson, 2018), donde los chicos en EF manifestaron sentir emociones negativas para su bienestar subjetivo (BS), ante la presión social de encajar en las conductas motrices de una masculinidad hegemónica. Por otro lado, diferentes investigaciones han reflejado que las chicas sienten emociones negativas para su BS ante contextos marcados por la cultura patriarcal, la poca comprensión de los problemas asociados al género y la falta de respeto hacia ellas (Azzarito et al., 2006; Camacho-Miñano y Aragón, 2014; Lodewyk y Muir, 2017; Monforte y Pérez-Samaniego, 2017; Timken et al., 2019). Frente a esta problemática, una revisión bibliográfica sobre el tema concluyó que, en ocasiones, el propio profesorado sería una fuente importante de reproducción de estereotipos de género (Alvariñas-Villaverde y Pazos-González, 2018).

Un segundo aspecto cultural que será abordado en este ensayo, es el del nivel socioeconómico y su relación con la cultura motriz de cada estudiante. Entre los grandes conflictos que se viven en occidente, en el marco de una cultura capitalista, está el de la pobreza, la concentración de las riquezas y la desigualdad sociocultural, lo cual, evidentemente, ha de tener diferentes consecuencias en EF. No obstante, como muestran diferentes revisiones bibliográficas (Bermúdez y Sáenz-López, 2019; Espoz-Lazo et al., 2020; Mujica, 2019; Mujica y Orellana, 2019; Mujica et al., 2017), es una línea de estudio en EF relativamente ausente desde la perspectiva emocional. Las causas sobre la invisibilización 
de la relación entre emociones y nivel socioeconómico en EF pueden ser variadas. En primer lugar, se señala que, en general, el estudio de las emociones en EF lleva desarrollándose poco tiempo. En segundo lugar, podría estar incidiendo, la todavía hegemónica mirada positivista de la investigación científica, la cual, usualmente, ha menospreciado la mirada crítica de las ciencias sociales que indaga diversos aspectos culturales. Por último, hemos de mencionar que la presencia en EF de lógicas de carácter tecnicista, biomédica, mecanicista y neoliberal, tampoco han favorecido el análisis de estos aspectos.

Una posible línea de investigación para el tema del nivel socioeconómico y las emociones del alumnado en EF podría desarrollarse en torno al aprendizaje deportivo. Algunos deportes pueden ser catalogados como elitistas, ya que su práctica está condicionada a infraestructuras que requieren de un alto coste económico y, por ende, no toda la población puede acceder a ellos. Por supuesto, dicho carácter variará en función de los diferentes continentes y naciones. Algunos de ellos pueden ser el golf, rugby, tenis, pádel, natación e, incluso, baloncesto. Es necesario recordar que existen territorios en los que las personas ni siquiera pueden acceder a una multicancha deportiva. Este contexto socioeconómico y cultural, en muchas realidades, puede incidir en las experiencias que cada estudiante haya tenido a lo largo de su historia de vida. Por ello, la presencia o ausencia de dichas experiencias deportivas podría suscitar diversos significados emocionales cuando se enfrenten a un contenido didáctico igual o similar. En este sentido, cabe señalar que "una emoción puede integrar múltiples significados subjetivos que, desde una amplia mirada, pueden relacionarse con factores culturales, éticos, psicológicos o biológicos" (Mujica-Johnson y Jiménez, 2020, p. 50). Además del factor socioeconómico, también se aprecian otros aspectos culturales que podrían dar lugar a líneas de investigación, relativamente, ausentes sobre la temática en EF. Por ejemplo, la cultura digital (videojuegos, redes sociales o educación a distancia) en la infancia y adolescencia; la cultura de inclusión educativa hacia personas con necesidades educativas especiales (trastornos del espectro autista, movilidad reducida, falta de visión o audición, etc.); interculturalidad; sedentarismo; y la ausencia o presencia de una religión desde diferentes perspectivas.

Otro aspecto cultural a reflexionar es la relación entre el contacto y las emociones en EF. Esta cuestión podría ser abordada desde distintas dimensiones (Salgado-López, 2020), si bien nosotros lo haremos 
únicamente desde la óptica cultural. Al hablar de contacto y cultura, debemos detenernos en los estudios de proxemia, iniciados por Hall a mediados del siglo XX (Hall, 1963) y, en el que se basarán gran cantidad de autores posteriores. Este autor estableció una serie de espacios personales, en función de los cuales las reacciones del individuo, tanto comportamentales como emocionales, son distintas. Ahora bien, las dimensiones de estos espacios y su uso varían de unas culturas a otras. El mismo Hall adelantaba ya que sus estudios se referenciaban a población norteamericana de origen anglosajón, y que serían diferentes, de aplicarse en el mundo latino (Hall, 1989). En sentido similar, el contacto, como situación de máxima proximidad entre dos cuerpos depende, además, de otros aspectos de índole personal (Hall, 2003), asimismo de las peculiaridades del contexto cultural (Hall, 1989). El campo de la Educación Física y el Deporte no es ajeno a las influencias de la cultura, en la que se inserta, en cuanto a los usos del contacto. Ahora bien, también es cierto que se acepta una mayor permisividad que en otros ámbitos sociales (Argyle, 1990). Así, es frecuente ver a jugadores de baloncesto o voleibol palmeándose el trasero como muestra de apoyo y felicitación, algo fuera de lugar en otros contextos de la cultura occidental. De todas formas, estos comportamientos no siempre son bien vistos. Así, Bodis (1999) relaciona el exceso de contacto no socialmente admisible, con la desaparición de la práctica del rugby por parte de las instituciones católicas de educación francesas, a partir de 1907.

Dentro de los estudios centrados en las prácticas motrices, la relación entre contacto, cultura y emociones ha sido establecida a partir de trabajos como los de Canales (2006), Rovira (2010) o Salgado-López (2014). En ellos, se muestra como el contacto genera tanto emociones placenteras como displacenteras, en función de ciertos factores como puede ser la confianza entre los individuos, el tipo de tarea a realizar, etc. Si nos centramos en las displacenteras, los dos primeros trabajos citados, enmarcados en las prácticas motrices expresivas y las prácticas introyectivas, respectivamente, mostraron que las emociones vergüenza y asco son algo frecuentes durante los procesos de enseñanza de las mismas. De forma similar, el citado trabajo de Salgado-López (2014) muestra también la importancia de estas emociones, esta vez en un contexto menos esperable, como es el de las prácticas motrices de colaboración-oposición. En este caso, frente a los anteriores, el miedo se muestra como una emoción fundamental a tener en cuenta, de cara 
a optimizar el proceso de enseñanza-aprendizaje de este tipo de prácticas motrices. Resumidamente se podría decir que entre las principales fuentes de emociones negativas asociadas al contacto, desde un punto de vista cultural, podemos encontrar: por un lado, los aspectos relacionados con el género; $\mathrm{y}$, por otro, los tabúes asociados a ciertas partes del cuerpo, el concepto de limpieza e higiene, etc. Ejemplo de lo inicial serían las emociones surgidas de los contactos que se pueden producir durante el desarrollo de prácticas motrices entre géneros. Muestra de lo siguiente serían las emociones relacionadas con contactos a zonas del cuerpo consideradas sexuales, o próximas a ellas; contactos con áreas corporales consideradas sucias en algunas culturas, como los pies; contactos con el suelo, que en la cultura occidental se tiende a asociar con fuente de gérmenes y enfermedades, etc.

\section{Aprendizaje significativo y emociones en Educación Física}

En este apartado, se realizarán diferentes reflexiones en torno al AS en EF, desde la perspectiva emocional y cultural, al considerar que el ser humano durante su aprendizaje no se encuentra fragmentado. Es decir, basado en una perspectiva fenomenológica es un cuerpo vivo (Leibkörper) (Husserl, 1996; Merleau-Ponty, 1993; Mujica, 2020), que se desarrolla en un contexto histórico-cultural concreto y, a su vez, posee su propio historial de vida que le ha ayudado a configurar diversos sentidos y significados en torno a la realidad. Precisamente, el concepto de conducta motriz de Parlebas (2001) se encuentra en esta línea argumentativa, al destacar los aspectos subjetivos y socioculturales. Antes de asociar los significados culturales del apartado anterior, con las emociones y el AS, pasaremos a revisar algunas premisas de esta última teoría de aprendizaje.

La teoría del AS se enfoca, principalmente, en el aprendizaje de corte intelectual (representaciones, proposiciones, conceptos, etc.), al hacer énfasis en la relación de los nuevos conocimientos o ideas, con los ya establecidos en la estructura cognitiva de cada aprendiz (Ausubel et al., 1983; Moreira, 2012). Por lo tanto, es preciso entender que dicha teoría no fue pensada para otro tipo de aprendizajes, por ejemplo, el de tipo motor, que es el principal en el área de EF. No obstante, esta puede ser transferida a ese último aprendizaje, sobre todo, si se considera que, en todo aprendizaje motor es fundamental el ámbito cognitivo y 
afectivo (Castejón y López-Ros, 2002; Mujica y Orellana, 2019; Parlebas, 1970, 2017; Salgado-López, 2014, 2020). De acuerdo con Ausubel et al. (1983), el AS se concreta cuando los estudiantes logran adquirir nuevos significados. Lo anterior sería resultado de la esencia del proceso que permite el AS; en concreto, que "las ideas expresadas simbólicamente son relacionadas de modo no arbitrario y sustancial (no al pie de la letra) con lo que el alumno ya sabe" (Ausubel et al., 1983, p. 48). En términos de motricidad, diríamos que las conductas motrices son asociadas de modo no arbitrario y sustancial con lo que la persona que aprende, ya sabe. Cabe destacar, que por no arbitrario y sustancial se hace referencia a que debe ser una asociación auténtica; por lo tanto, contraria a una relación mecánica o automatizada.

Con respecto al ámbito actitudinal, motivacional y emocional del aprendizaje, Ausubel et al. (1983) plantearon que, son importantes las experiencias agradables o placenteras para potenciar el aprendizaje. Sobre todo, porque son una importante fuente de motivación intrínseca y, por ende, propicia una disposición o actitud favorable hacia su contenido. Dicha actitud sería muy importante en diferentes funciones ejecutivas, por ejemplo, en la capacidad de mantener la atención en el objeto de aprendizaje. En este sentido, señalaron que cuando las actitudes "hacia un material de controversia son favorables, los sujetos están muy motivados para aprender, despliegan esfuerzos más intensos y concentrados" (Ausubel et al., 1983, p. 371). Sin embargo, no es una condición sine qua non para que se produzca el AS, es decir, que sin esas experiencias también se podría producir. En otras palabras, es fundamental que el alumnado tenga disposición a aprender (con o sin gusto o agrado por el aprendizaje), como sucede en los casos en que las personas deciden aprender por una necesidad (Moreira, 2012). La lógica que sostienen estos autores radica en que, en el caso que no exista la motivación intrínseca, el propio AS la puede suscitar por medio de la satisfacción de haber logrado un sentido y significado al aprendizaje. En palabras de los autores aludidos, diríamos que "el aprendizaje significativo, a diferencia de otras clases de aprendizaje humano, proporciona automáticamente su propia recompensa" (Ausubel et al., 1983, p. 350).

En el marco de un AMS, Castejón y López-Ros (2002) han señalado que el AS contribuye a que los estudiantes puedan tener una mejor comprensión en torno a la funcionalidad de lo que se aprende, lo cual fue explicado de esta forma: 
... el alumno atribuye un sentido más profundo y razonable a su aprendizaje si comprende para qué sirve y por qué es necesario aprender un contenido determinado. Por ejemplo, el alumno que aprende el "bote de progresión" en baloncesto entendiendo que esa acción le va a servir para rebasar a un oponente o para avanzar hacia la canasta. (Castejón y López-Ros, 2002, p. 47)

En síntesis, para el AMS en EF, desde la perspectiva emocional, convendría tener en cuenta las siguientes premisas: a) las emociones placenteras pueden favorecer o incentivar el AMS; b) las emociones displacenteras pueden desfavorecer o desincentivar el AMS; c) el AMS puede concretarse con la presencia de emociones placenteras y displacenteras. Para esto, deben existir dos condiciones fundamentales; la primera es que exista disposición a aprender y, la segunda, es que el alumnado pueda conectar el contenido de aprendizaje, potencialmente significativo, con sus propios significados motrices; y d) el logro de AMS puede suscitar emociones placenteras como recompensa al esfuerzo realizado.

\section{Cultura y emociones en Educación Física en función del aprendizaje motor significativo}

Una vez vista la relación de la cultura con las emociones y, de estas últimas con el AMS, conviene realizar la triangulación entre estos diferentes conceptos abordados. Comenzaremos al señalar que existe una relación recíproca y cíclica entre los tres aspectos abordados, la cual, por su complejidad, no será ordenada jerárquicamente. En este sentido, solo acotaremos que los significados culturales inciden en las emociones y estas en los AMS, como se puede apreciar en la Figura 1. 


\section{Figura 1}

Relación recíproca y cíclica entre emociones, cultura y aprendizaje motor significativo
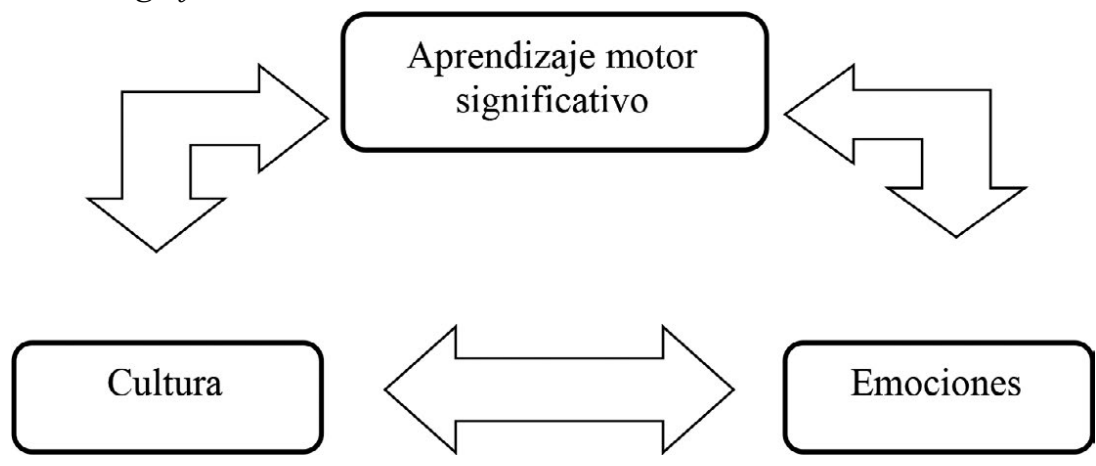

Nota: Elaboración propia de los autores.

Retomando los significados culturales planteados con anterioridad, se propondrán algunas aplicaciones prácticas que puedan contribuir a favorecer la motivación intrínseca desde la perspectiva emocional y, por ende, la actitud favorable de los estudiantes.

Desde la perspectiva de género, el profesorado debe tener en consideración que, ante dificultades de aprendizaje en una práctica motriz, por parte de una alumna o un alumno, cabe la posibilidad de que los estereotipos de género estén incidiendo en el proceso. Por ejemplo, si una chica se ha desarrollado en un entorno cultural que poco o nada le ha promovido la práctica de un deporte como fútbol, es comprensible que, ante el aprendizaje de dicho deporte como contenido didáctico, perciba poco o nada de emociones placenteras y muestre poco interés por él. De forma similar, tendríamos otro ejemplo para un chico que enfrente el contenido de gimnasia rítmica o de danza, disciplinas que dentro de la cultura occidental están tradicionalmente atribuidas al género femenino. Por otro lado, hemos de recordar que un alumno o una alumna también podrían presentar una mala disposición emocional frente a un contenido didáctico que, en su entorno cultural, haya estado marcado por la reproducción de estereotipos que no eran de su agrado. Por ejemplo, un chico que no le agrade la excesiva competitividad que se da en el fútbol u otro deporte. En el caso de una chica, estaría uno que, para ella, se asocie a un significado cultural de feminidad hegemónica (preocupación por la 
estética o distanciamiento de la competitividad). En cualquier caso, de los múltiples significados culturales asociados a los estereotipos de género que pueden presentarse en estudiantes durante las sesiones de EF, es muy importante que el profesorado proponga objetivos que amplíen los significados y trasciendan los estereotipos hegemónicos.

Sensibilizar a los estudiantes con la perspectiva de género puede permitirles visualizar manifestaciones motrices alternativas a las hegemónicas y, demostrarles que no existe una sola forma de ser y actuar, menos asociada al género. A modo de ilustración, entre muchos otros casos, se les puede narrar la historia de Katherine Switzer, la primera mujer que, al infringir las reglas establecidas, corrió una maratón en 1967 en Boston, Estados Unidos. De este modo, rompió el estereotipo de que ellas no lo podían hacer y generó una transformación mundial en la práctica del atletismo. En este sentido, sería constructivo que el profesorado reconozca y comprenda las emociones displacenteras asociadas a significados culturales en torno al género. Las cuales, además, no favorecerían el AS. Sin embargo, dichas emociones tendrían raíces muy profundas, de modo que para los estudiantes no sería una tarea sencilla superarlas. Por lo mismo, desde el quehacer docente se podrían utilizar variadas estrategias para promover el AMS del alumnado, considerando que existirían dificultades en la disposición emocional. Por ejemplo, refuerzo positivo, metodologías innovadoras, paciencia ante las dificultades de los aprendices y, por, sobre todo, coeducación desde perspectivas de género alternativas a las patriarcales.

En función del nivel socioeconómico, cabrían diversos problemas en los significados culturales. En los entornos con mayor pobreza podría ser que exista una desvinculación muy profunda con algunas prácticas motrices, en comparación a otros entornos más favorecidos desde la perspectiva socioeconómica. Esta carencia de experiencias y, por ende, de significados motrices, podrían dificultar la disposición emocional, aunque, como se ha mencionado antes, es un tema que requiere ser más investigado. Para reflexionar sobre estos contextos, pondremos un ejemplo en correspondencia complejo. El caso de una estudiante que en sus 14 años de vida no ha podido tener experiencias con la natación y que tiene que enfrentarse a un aprendizaje motor en dicho contenido didáctico. Seguramente, frente a este caso podríamos encontrarnos con una alumna que siente emociones displacenteras y, en consecuencia, poca disposición emocional. Por consiguiente, sería fundamental 
coconstruir poco a poco significados motrices y, de este modo, aproximarse, de forma pausada, a una percepción de seguridad y entusiasmo, frente a este nuevo contexto de aprendizaje motor. No obstante, en el aprendizaje de natación en edades avanzadas, también podrían existir otros importantes factores que inciden en la aparición de este tipo de emociones y que son independientes del factor socioeconómico.

De acuerdo con el contacto, al seguir el trabajo de Salgado-López (2020), cabría distinguir los contactos que se producen entre individuos, móviles y suelo. En el citado trabajo se realiza un estudio pormenorizado de los factores que cabría valorar para optimizar los procesos de aprendizaje de prácticas motrices que impliquen contacto. Sintetizando la extensa propuesta que se presenta podemos decir que: a) en los casos de situaciones de aprendizaje de prácticas motrices que susciten miedo vinculado al contacto, se deben extremar las situaciones de seguridad (reducir la velocidad de contacto, la altura de caída, utilizar superficies amortiguantes para las caídas, facilitar el conocimiento de los materiales a usar antes de la práctica, etc.); b) las situaciones motrices en las que los sujetos puedan sentir vergüenza asociada a los contactos que se producen en la tarea (principalmente interpersonales), cabe maximizar los procesos de socialización del grupo, al hacer reflexión crítica de los tabúes y estereotipos sociales que suelen estar en el origen de esa emoción; c) por último, en cuanto a la emoción asco, relacionadas con aspectos higiénicos de las superficies a contactar o con tabúes y estereotipos sociales de ciertas partes del cuerpo, cabrían intervenciones similares a las mostradas para el caso anterior.

\section{Consideraciones finales}

A lo largo de este trabajo, hemos intentado mostrar, de forma resumida, cómo los significados culturales pueden favorecer o entorpecer el AMS de los estudiantes, ya que inciden en la disposición emocional y la actitud de los estudiantes. En el caso de que existan significados culturales que promuevan emociones displacenteras ante algún contenido didáctico en $\mathrm{EF}$, podría ser necesario favorecer la adquisición de nuevos significados que tengan una orientación contraria. Esto, probablemente beneficiaría la disposición emocional de los estudiantes. De forma precisa, sería muy beneficioso que se desarrolle una línea de investigación en torno a dichas intervenciones didácticas. 
De acuerdo con la teoría del AS, las emociones displacenteras podrían ser superadas a medida que el alumnado vaya adquiriendo nuevos significados, en el caso de la EF, nuevas conductas motrices. Por lo tanto, la disposición emocional podría mejorar, sustancialmente, en función del éxito de los AMS. Desde la perspectiva cultural, consideramos que es importante entender que algunos de sus significados pueden estar muy enraizados en la configuración subjetiva de los estudiantes, de modo que apreciamos que valores como la paciencia, la perseverancia y la constancia tendrían un rol relevante en dicho proceso pedagógico. Además, el sentido crítico frente a las corrientes dominantes y la capacidad de análisis, para poder discernir cuáles son esas influencias culturales en el aprendizaje.

\section{Referencias}

Altieri, A. (2001). ¿Qué es cultura? La Lámpara de Diógenes, 2(2), 15-20.

Alvariñas, M., Fernández-Villarino, M. A. y López-Villar, C. (2009). Actividad física y percepciones sobre deporte y género. Revista de Investigación en Educación, 6, 113-122. Recuperado de https://dialnet.unirioja.es/descarga/articulo/3216408.pdf

Alvariñas-Villaverde, M. y Pazos-González, M. (2018). Estereotipos de género en Educación Física, una revisión centrada en el alumno. Revista Electrónica de Investigación Educativa, 20(4), 154-163. https://doi.org/10.24320/redie.2018.20.4.1840

Argyle, M. (1990). Bodily Communication. Routledge.

Ausubel, D. (2002). Adquisición y retención del conocimiento: una perspectiva cognitiva. Paidós.

Ausubel, D., Novak, J. y Hanesian, H. (1983). Psicología Educativa. Un punto de vista cognoscitivo (2. ${ }^{\mathrm{a}}$ ed.). Trillas.

Azzarito, L., Solmon, M. y Harrison, L. (2006). “...If I Had a Choice, I Would....". A Feminist Poststructuralist Perspective on Girls in Physical Education. Research Quarterly for Exercise and Sport, 77(2), 222-239. https://doi.org/10.1080/02701367.2006.10599356 Bermúdez, C. y Sáenz-López, P. (2019). Emociones en Educación Física. Una revisión bibliográfica (2015-2017). Retos. Nuevas Tendencias en Educación Física, Deportes y Recreación, 36, 597603. https://doi.org/10.47197/retos.v36i36.70447 
Bodis, J.P. (1999). Les catholiques et le Rugby, en France et ailleurs: des attitudes contratées. En: G. Cholvy e Y. Tranvouez, (Eds.), Sport, culture et religion. Les patronages catholiques (18981998), 201-209. Centre de Recherche Bretonne et Celtique. Université de Bretagne Occidentale.

Bourdieu, P. (2000). La dominación masculina. Anagrama.

Bozalek, V. y Zembylas, M. (2016). Critical posthumanism, new materialisms and the affective turn for socially just pedagogies in higher education. South African Journal of Higher Education, 30(3), 193-200. https://www.journals.ac.za/index.php/sajhe/ article/view/652

Bruner, J. (1997). La educación, puerta de la cultura. Visor.

Cáceres, Z. y Munévar, O. (2016). Evolución de las teorías cognitivas y sus aportes a la educación. Revista Actividad Física y Desarrollo Humano, 7, 1-13. https://doi.org/10.24054/16927427. v2.n2.2016.2408

Camacho-Miñano, M. (2013). Contradicciones del significado de la actividad físico-deportiva en las identidades corporales de las chicas adolescentes. Feminismo/s, 21, 15-35. https://doi.org/10.14198/ fem.2013.21.02

Camacho-Miñano, M. y Aragón, N. (2014). Ansiedad física social y educación física escolar: las chicas adolescentes en las clases de natación. Apunts. Educación Física y Deportes, 116(2), 87-94. https://doi.org/10.5672/apunts.2014-0983.es

Canales, I. (2006). Consecuencias pedagógicas de la mirada y el tacto en la expresión corporal (Tesis doctoral no publicada). Universitat de Lleida. Lleida. http://hdl.handle.net/10803/8205

Castejón, F. J. y López-Ros, V. (2002). Consideraciones metodológicas para la enseñanza y el aprendizaje del deporte escolar. Tándem. Didáctica de la Educación Física, 7, 42-55. https://dialnet.unirioja.es/servlet/articulo? codigo $=237538$

Espoz-Lazo, S., Rodríguez, R., Espoz-Lazo, P., Farías-Valenzuela, C. y Valdivia-Moral, P. (2020). Emotional Education for the Development of Primary and Secondary School Students Through Physical Education: Literature Review. Education Sciences, 10(192), 1-12. https://doi.org/10.3390/educsci10080192

Foucault, M. (2019). Microfisica del poder. Siglo XXI. 
Gaviria, D. y Castejón, F. J. (2016). Desarrollo de valores y actitudes a través de la clase de Educación Física. Movimento, 22(1), 251262. https://doi.org/10.22456/1982-8918.53455

Gea-García, G. M., Alonso-Roque, J. I., Yuste Lucas, J. L. y Garcés de los Fayos-Ruiz, E. J. (2016). Los juegos deportivos y su influencia en la gestión emocional en universitarios. Cuadernos de Psicología del Deporte, 16(3), 101-112. https://revistas.um.es/cpd/ article/view/278511

Gerdin, G. y Larsson, H. (2018). The productive effect of power: (dis) pleasurable bodies materialising in and through the discursive practices of boys' physical education. Physical Education and Sport Pedagogy, 23(1), 66-83. https://doi.org/10.1080/1740898 9.2017.1294669

González, F. (2009). La significación de Vygotski para la consideración de lo afectivo en la educación: bases para la cuestión de la subjetividad. Revista Electrónica Actualidades Investigativas en Educación, 9, 1-24. https://www.redalyc.org/pdf/447/44713052003.pdf

Hall, E. T. (1963). A System for the Notation of Proxemic Behavior. American Anthropologist, 65(5), 1003-1026. http://www.jstor. org/stable $/ 668580$

Hall, E.T. (1989). El lenguaje silencioso. Alianza Editorial.

Hall, E.T. (2003). La dimensión oculta. Siglo XXI.

Heinemann, K. y Puig, N. (1997). Emocions en l'esport. Esbós d'una anàlisi sociològica. Revista Catalana de Sociología, 5, 117146. https://www.raco.cat/index.php/RevistaSociologia/article/ view/14601/318481

Husserl, E. (1996). Meditaciones cartesianas. Fondo de Cultura Económica.

Lagardera, F. (1999). La lógica deportiva y las emociones. Sus implicaciones en la enseñanza. Apunts. Educación Física y Deportes, 56, 99-106. https://www.raco.cat/index.php/ApuntsEFD/article/ view/307029

Lavega, P., Filella, G., Agulló, M.J., Soldevila, A. y March, J. (2011). Conocer las emociones a través de juegos: Ayuda para los futuros docentes en la toma de decisiones. Electronic Journal of Research in Educational Psychology, 9(24), 617-640. https://doi. org/10.25115/ejrep.v9i24.1459

Lévi-Strauss, C. (1971). Arte, Lenguaje y Etnología (3. ${ }^{a}$ ed.). Siglo XXI. 
Lévi-Strauss, C. (2019). Raza y cultura (9. a ed.). Cátedra.

Lodewyk, K. y Muir, A. (2017). High school females' Emotions, self-efficacy, and attributions during soccer and fitness testing in Physical Education. Physical Educator, 74(2), 269-295. https:// doi.org/10.18666/TPE-2017-V74-I2-7136

Merleau-Ponty, M. (1993). Fenomenología de la percepción. Planeta-Agostini.

Miralles, R., Filella, G. y Lavega, P. (2017). Educación Física emocional a través del juego en educación primaria. Ayudando a los maestros a tomar decisiones. Retos. Nuevas Tendencias en Educación Física, Deporte y Recreación, 31, 88-93. https://www.redalyc.org/pdf/3457/345750049016.pdf

Monforte, J. y Pérez-Samaniego, V. (2017). El miedo en Educación Física: una historia reconocible. Movimento, 23(1), 85-99. https:// doi.org/10.22456/1982-8918.71272

Moreira, M. A. (2012). ¿Al final, qué es Aprendizaje significativo? Revista Qurriculum, 25, 29-56. https://riull.ull.es/ xmlui/bitstream/handle/915/10652/Q_25_\%282012\%29_02. pdf? sequence $=5 \&$ isAllowed $=\mathrm{y}$

Mujica, F. (2020). El término Educación Física en la posmodernidad: contribución de algunas perspectivas fenomenológicas. Retos. Nuevas Tendencias en Educación Física, Deportes y Recreación, 38, 795-801. https://doi.org/10.47197/retos.v38i38.73011

Mujica-Johnson, F. y Jiménez, A. C. (2020). Percepción emocional del alumnado de $3^{\circ}$ de ESO ante las prácticas de la unidad didáctica de baloncesto en Educación Física. Revista Española de Educación Física y Deportes, 429, 47-60. https://www.reefd.es/index. php/reefd/article/view/899/745

Mujica, F. y Orellana, N. (2019). Emociones en educación física desde la perspectiva constructivista: análisis de los currículos de España y Chile. Praxis \& Saber, 10(24), 297-319. https://doi. org/10.19053/22160159.v10.n25.2019.8468

Mujica, F., Orellana, N. y Concha, R. (2017). Emociones en la clase de Educación Física: revisión narrativa (2010-2016). Ágora para la Educación Física y el Deporte, 19(1), 119-134. https://doi. org/10.24197/aefd.1.2017.119-134

Mujica, F., Orellana, N. y Luis-Pascual, J. C. (2019). Perspectiva moral de las emociones en los contextos de educación formal. Revista 
Ensayos Pedagógicos, 14(1), 69-90. https://doi.org/10.15359/ rep.14-1.4

Parlebas, P. (1970). L'affectivité, clef des conduites motrices. EPS, 102, 70-74.

Parlebas, P. (2001). Juegos, deportes y sociedades. Léxico de praxiología motriz. Paidotribo.

Parlebas, P. (2017). La aventura praxiológica. Ciencia, acción y educación fisica. Consejería de Turismo y Deporte.

Piaget, J. (1991). Seis estudios de psicología. Labor.

Puig, N. (2012). Emociones en el deporte y sociología. Revista Internacional de Ciencias del Deporte, 8(28), 106-108. https://doi. org/10.5232/ricyde2012.028

Ron, J. (1977). Sobre el concepto de cultura. Instituto Andino de Artes Populares.

Rovira, G. (2010). La conciencia sensitiva en la formación del docente. Estudio de casos: Las vivencias de los estudiantes de magisterio suscitadas en la práctica de situaciones motrices introyectivas [Tesis de doctoral no publicada]. Universidat de Lleida, Lleida.

Sáez de Ocáriz, U., Lavega, P., Lagardera, J., Costes, A. y Serna, J. (2014). ¿Por qué te peleas? Conflictos motores y emociones negativas en la clase de Educación Física: el caso de los juegos de oposición. Educatio Siglo XX1, 32(2), 71-90. https://doi. org/10.6018/j/194091

Salgado-López, J. I. (2014). As emoções como condicionante didático no ensino dos deportes sociomotores de colaboração-oposição: estudo dos problemas afetivos ligados ao contacto en rugby, andebol e voleibol [Tesis doctoral no publicada]. Universidade da Coruña, A Coruña.

Salgado-López, J. I. (2020). Contacto y emociones en la educación fisica y el deporte. Factores didácticos para optimizar un aprendizaje inclusivo. Sevilla: Wanceulen.

Scheler, M. (2010). Amor y conocimiento. Y otros escritos. Palabra.

Timken, G., McNamee, J. y Sarah, C. (2019). 'It doesn't seem like PE and I love it': Adolescent girl's views of a health club physical education approach. European Physical Education Review, 25(1), 1-16. https://doi.org/10.1177/1356336X17706382

Viera, T. (2003). El aprendizaje verbal significativo de Ausubel. Algunas consideraciones desde el enfoque histórico-cultural. 
Revista Universidades, 26, 37-43. https://www.redalyc.org/ pdf/373/37302605.pdf

Vygotsky, L. (1995). Pensamiento y lenguaje. Teoría del desarrollo cultural de las funciones psíquicas. Fausto.

Zembylas, M. (2019). Intentos por discernir la compleja imbricación entre emoción y pedagogía: contribuciones del giro afectivo. Propuesta Educativa, 28(51), 15-29. https://www.redalyc.org/ jatsRepo/4030/403061372003/html/index.html 